\title{
Listeria monocytogenes Meningitis in an Immunocompetent Adult Patient
}

\author{
W.Y. Jamala S. Al-Shomari ${ }^{b}$ F. Boland ${ }^{a}$ V.O. Rotimi ${ }^{a, c}$ \\ a Department Microbiology, Mubarak Al-Kabeer Hospital, b Department of Medicine, Faculty of Medicine and \\ Mubarak Hospital, and ' Department of Microbiology, Faculty of Medicine, Kuwait University, Kuwait
}

\section{Key Words}

Listeria monocytogenes . Meningitis .

Immunocompetent adult
L. monocytogenes in a previously healthy young adult with no risk factor. Only a few similar cases have been reported in the literature.

Copyright @ 2005 S. Karger AG, Basel

\begin{abstract}
Objective: To report an interesting case of meningitis caused by Listeria monocytogenes in an immunocompetent adult. Patient and Methods: A previously healthy 25year-old man presented with typical clinical features of meningitis. Cerebrospinal fluid (CSF) was obtained on the day of admission for biochemical and microbiological investigations. In addition, blood was also taken for culture and hematological studies. Antibiotic susceptibility test was performed using the Etest method. Microscopic examination of the CSF showed pleocytosis, which was predominantly lymphocytic, while the biochemical investigation revealed raised concentrations of protein and lactic acid as well as decreased glucose concentration. A 24-hour culture yielded pure growth of gram-positive bacilli identified by standard methods as L. monocytogenes. It was susceptible to ampicillin and trimethoprim-sulfamethoxazole. The patient was treated with intravenous ampicillin combined with gentamicin and made a complete recovery. Conclusion: This presentation describes an unusual case of meningitis caused by
\end{abstract}

\section{Introduction}

Listeria monocytogenes is a gram-positive, facultative anaerobic, motile bacillus that has a characteristic tumbling motility at $20-25^{\circ} \mathrm{C}$. It produces $\beta$-hemolysis on blood agar. Its infection in humans is not very common, but it has become increasingly important in neonatal infections and infections in immunocompromised individuals. It also causes infections in many different animal species including cattle, pigs, rodents, fowls, and fish. Outbreaks and sporadic episodes of listeriosis are usually food-borne such as milk, cheese, coleslaw, meat products or vegetables. Asymptomatic intestinal carrier state exists in about $1-5 \%$ of humans, which provides a reservoir for this pathogen [1].

L. monocytogenes is becoming an important cause of community-acquired acute meningitis [2]. The meningitis it causes differs from the other types of meningitis in epidemiology, clinical features, cerebrospinal fluid (CSF) findings, treatment and prognosis. The organism is intrin-

\begin{tabular}{ll}
\hline KARGER & ( ) 2005 S. Karger AG, Basel \\
Fax +4161306 1234 & 1011-7571/05/0141-0055\$22.00/0 \\
$\begin{array}{l}\text { E-Mail karger@karger.ch } \\
\text { www.karger.com }\end{array}$ & $\begin{array}{l}\text { Accessible online at: } \\
\text { www.karger.com/mpp }\end{array}$
\end{tabular}

Prof. V.O. Rotimi

Department of Microbiology, Faculty of Medicine

Kuwait University, PO Box 24923

13110 Safat (Kuwait)

Tel. +965 531 2300, ext.6509, Fax +965 533 2719, E-Mail vincent@hsc.edu.kw 
sically resistant to the third-generation cephalosporins that are used empirically for the treatment of bacterial meningitis. In addition, any delay in initiating appropriate therapy may lead to a poor outcome with high mortality rate, which may reach up to $30 \%$ [3].

It is important that the clinician should be familiar with the features of this infection in order to recognize and manage it successfully. The aim of this study was to report a case of meningitis caused by $L$. monocytogenes in a previously healthy immunocompetent adult patient.

\section{Case Report}

A 25-year-old healthy Kuwaiti man was admitted to Mubarak AlKabeer Hospital, Kuwait on March 22, 2003 because of a 1-day history of fever, headache and vomiting without any predisposing underlying medical problem. His past medical history was nonsignificant.

On examination, he was ill-looking, sweaty but well oriented. $\mathrm{He}$ was febrile (oral temperature: $38.5^{\circ} \mathrm{C}$ ). There was neck stiffness with positive Kernig's sign. Other examinations of the central nervous system (CNS), respiratory, cardiovascular and abdominal systems were normal.

The initial laboratory investigations were the following: WBC $19.6 \times 10^{9} / 1$, RBC $4.5 \times 10^{9} / 1$, platelets $182 \times 10^{9} / 1$. His serum electrolytes, liver and renal function tests were all normal. CT scan of the brain was also normal. CSF was obtained via a lumbar puncture and sent to the laboratory for microbiological and biochemical investigations. Microscopy showed pleocytosis (WBC 922/ $\mathrm{mm}^{3}$; lymphocytes $75 \%$ and polymorphs $25 \%$ ). The protein content was high (1,665 mg/l; normal range $150-450 \mathrm{mg} / \mathrm{l})$, LD $148 \mathrm{IU} / \mathrm{l}$, glucose level slightly reduced $(2.6 \mathrm{mmol} / \mathrm{l}$; serum glucose $6.6 \mathrm{mmol} / \mathrm{l})$. Gram stain and Ziehl-Neelsen stain of the CSF smear did not demonstrate any microorganism. Latex agglutination (Directigen ${ }^{\mathrm{TM}}$, Becton Dickinson Microbiology Systems, Sparks, Md., USA) was negative for Neisseria meningitidis, Haemophilus influenzae and Streptococcus pneumoniae. India ink preparation for Cryptococcus spp. was negative.

The patient was treated initially with an antimeningitic dose of intravenous (i.v.) ceftriaxone, $2 \mathrm{~g}$ twice daily. On day 2 after admission, the patient was still febrile but neurological examination was normal except for persistent stiff neck and positive Kernig's sign. The culture of the CSF yielded a growth of gram-positive bacilli later identified as L. monocytogenes susceptible to penicillin, ampicillin, gentamicin, teicoplanin, vancomycin and tetracycline by the Etest (AB Biodisk, Slona, Sweden). The minimum inhibitory concentrations of penicillin and ampicillin were 0.19 and $0.25 \mu \mathrm{g} / \mathrm{ml}$, respectively. Consequently, his therapy was changed to i.v. ampicillin $2 \mathrm{~g}$ 4-hourly plus gentamicin $120 \mathrm{mg}$ 8-hourly. The patient remained febrile with headache for the next $24 \mathrm{~h}$. However, there was defervescence on day 6 with marked clinical improvement. The patient's blood culture remained negative after 7 days' incubation. After 10 days of treatment he was well and discharged home.

\section{Discussion}

The reported incidence of listerial meningitis among all episodes of community-acquired acute meningitis among adults in North America and Europe is from 3 to $10 \%$ [3-5]. It affects males twice as often as females and usually very young, elderly and immunocompromised individuals [6]. It is clinically prudent to exercise a high degree of suspicion in young children less than 3 years old and adults more than 50 years old that present with meningitis with no clear etiology. Our patient was a healthy immunocompetent young man who was neither a farmer nor engaged in animal husbandry-related work. Thus listerial meningitis was not suspected. It was difficult to pinpoint the source of his infection. It is likely that he was just an asymptomatic intestinal carrier as the incidence in carriers ranges between 1 and $5 \%$ of the population [1].

To the best of our knowledge, our patient was healthy prior to his illness and did not appear to have any apparent food-related source for his infection. It is important to emphasize that even in large specialized centers that provide care for a large number of immunocompromised patients, the clinical experience with cerebral listeriosis and therapeutic options are limited. Unlike the traditional bacterial etiological agents of meningitis (i.e. S. pneumoniae, H. influenzae and N. meningitidis), L. monocytogenes has tropism for the human brain parenchyma as well as the meninges [7]. Patients with meningitis may present with fever, headache, nausea, vomiting, neck stiffness and seizures. These patients are clinically indistinguishable from those with the more common etiological agents of meningitis. However, some clinical differences exist. The incidence of meningeal signs among patients with L. monocytogenes is lower than that among cases of meningitis due to other causes of bacterial meningitis, which in fact may reach statistical significance $[3,8]$. Therefore, it is noteworthy that the absence of meningeal signs does not exclude listerial meningitis. However, many patients with $L$. monocytogenes infection have a presentation more consistent with meningoencephalitis including altered consciousness and movement disorders [7]. L. monocytogenes may rarely cause other forms of CNS infections. For instance, cerebritis, representing the earliest form of intracranial inflammation before the observation of microscopic pus, has been seen especially in patients with organ transplantation [9]. A peculiar infection of the brain stem by L. monocytogenes, termed rhombencephalitis, should be suspected when asymmetric cranial nerve palsies, encephalopathy, cerebellar signs, hemiparesis or hypesthesia follows nonspecific symptoms 
of fever and headache [10]. Brain abscesses may be seen in up to $10 \%$ of listerial CNS infections, often as a complication of clinical meningitis.

Evidence in this case showed that the CSF finding was slightly atypical (lymphocytic pleocytosis) for common bacterial meningitis, even though he had typical signs and symptoms of meningitis, and the gram-stained smear did not demonstrate a causative organism. Even when the cultures later revealed the characteristic gram-positive rods, they could have been mistaken for contaminating 'diphtheroids' and the listeria might not have been reported but for the vigilance of the experienced technical staff.

The optimal treatment of CNS listeriosis is still uncertain and there are no prospective, controlled trials in an adequate number of patients. Our patient was treated with ampicillin and gentamicin and he made full recovery although defervescence after initiating this combination therapy took 4 days. Ampicillin or penicillin is the most widely used antibiotic for treating listerial infections. A few microbiological data have suggested that ampicillin may be superior to penicillin for treating CNS listeriosis [11]. Both in vitro and in vivo data have documented a synergistic effect when aminoglycoside is added to ampicillin or penicillin [10]. Furthermore, the addition of aminoglycoside has been found to reduce the mortality rate and improve the efficacy of ampicillin or penicillin [3]. The third-generation cephalosporins, despite their broad spectrum of activity in general, are poorly active against Listeria spp. and are not acceptable choices for treatment of listeriosis. Trimethoprim-sulfamethoxazole seems to have bactericidal activity against $L$. monocytogenes. Therefore, in patients with severe allergy to $\beta$-lactam antibiotic, who cannot be desensitized, trimethoprim-sulfamethoxazole may be the best alternative. Treatment with tetracycline, erythromycin, vancomycin and chloramphenicol has also been associated with failure [12]. All these findings point to a limited therapeutic option for the treatment of CNS listeriosis. Another important point of note in the treatment of CNS listeriosis is the duration of treatment. Because of the prolonged period of treatment required to kill the intracellular organism, extended time of defervescence and the high relapse rate when the patient is treated for less than 2 weeks, it is suggested that the duration of the treatment for CNS listeriosis should be at least 2-3 weeks. In cases of complications with microabscesses, particularly among patients with focal neurological findings, the duration should even be longer [4]. Although listerial infection is more common in neonates, elderly and immunocompromised patients, it can occur in previously healthy nonimmunosuppressed patients.

\section{Conclusion}

This presentation describes an unusual case of meningitis caused by $L$. monocytogenes in a previously healthy young adult with no risk factor. Only a few similar cases have been reported in the literature.

\section{References}

$\checkmark 1$ Embil JA, Ewan EP, McDonald SW: Surveillance of Listeria monocytogenes in human and environmental specimens in Nova Scotia, 1974 to 1981. Clin Invest Med 1984;7:325-327.

$\checkmark 2$ Durand ML, Calderwood SB, Weber DJ, Miller SI, Southwick FS, Caviness VS Jr, Swartz MN: Acute bacterial meningitis in adults: A review of 493 episodes. N Engl J Med 1993; 328:21-28.

3 Mylonakis E, Hohmann EL, Calderwood SB: Central nervous system infection with Listeria monocytogenes: 33 years experience at a general hospital and review of 776 episodes from the literature. Medicine 1998;77:313-336.

4 Campbell DM: Human listeriosis in Scotland, 1967-1988. J Infect 1990;20:241-250.
5 Schuchat A, Robinson K, Wenger JD, Harrison LH, Farely M, Reingold AL: Bacterial meningitis in the United States in 1995. N Engl J Med 1997;337:970-976.

-6 Davey PG, Cruikshank JK, McManus IC, Mahood B, Snow MH, Geddes AM: Bacterial meningitis -10 years experience. J Hyg (Lond) 1982;88:383-401.

7 Neiman RE, Lorber B: Listeriosis in adults: A changing pattern. Report of eight cases and review of the literature, 1968-1978. Rev Infect Dis 1980;2:207-221.

8 Tunkel AR, Scheld WM: Acute meningitis; in Mandel GL, Bennett JE, Dolin R (eds): Principle and Practice of Infectious Diseases, ed 4. New York, Churchill Livingstone, 1995, pp 831-865.
-9 Watson GW, Fuller TJ, Elms J, Kluge RM: Listeria cerebritis: Relapse of infection in renal transplant patients. Arch Intern Med 1978; 138:83-87.

10 Armstrong RW, Fung PC: Brainstem encephalitis (rhombencephalitis) due to Listeria monocytogenes: Case report and review. Clin Infect Dis 1993;16:689-702.

11 Jones EM, MacGowan AP: Antimicrobial chemotherapy of human infection due to Listeria monocytogenes. Eur J Clin Microbiol Infect Dis 1995; 14:165-175.

12 Baldassarre JS, Ingerman MJ, Nansteel J, Santoro $\mathrm{J}$ : Development of listerial meningitis during vancomycin therapy: A case report (letter). J Infect Dis 1991;164:221-222. 